Publicacions Matemàtiques, Vol 37 (1993), 355-358.

\title{
DUAL DIMENSION OF MODULES OVER NORMALIZING EXTENSIONS
}

\author{
AHMAD SHAMSUDDIN
}

Abstract

Let $S=\sum_{i=1}^{n} R a_{2}$ be a finite normalizing extension of $R$ and suppose that $S M$ is a left $S$-module. Denote by $\operatorname{crk}(A)$ the dual Goldie dimension of the module $A$. We show that $\operatorname{crk}\left({ }_{R} M\right) \leq$ $n \cdot \operatorname{crk}(s M)$ if either $s M$ is artinian or the group homomorphism $M \rightarrow a_{i} M$ given by $x \mapsto a_{2} x$ is an isomorphism.

1. Let $R$ be a ring and let $M$ be a left $R$-module. The Goldie dimension of $M$, defined as the cardinality of a maximal independent family of submodules of $M$, is denoted by $\operatorname{rk}(M)$. A family $A_{1}, \ldots, A_{n}$ of proper submodules of $M$ is said to be coindependent if for each index $i, 1 \leq i \leq n, A_{i}+\bigcap_{3 \neq i} A_{j}=M$. A family $\left(A_{i}\right)_{i \in I}$ of submodules of $M$ is said to be coindependent if each of its finite subfamilies is coindependent. The module $M$ is said to be hollow if $M \neq 0$ and if every proper submodule of $M$ is superfluous in $M$. Every family of submodules of $M$ contains a maximal coindependent subfamily. The cardinality of a maximal coindependent family of submodules of $M$, denoted by $\operatorname{crk}(M)$, is called the dual Goldie dimension of $M$. We shall need the following results, which can be found in [2], [3], [6].

1.1. If $N$ is a proper submodule of $M$ and if crk is finite then there exists a finite family of submodules $\left(A_{i}\right)_{i \in I}$ of $M$ such that $\{N\} \cup$ $\left\{A_{i}: i \in I\right\}$ is coindependent, $M / A_{i}$ is hollow for each $i \in I$, and $N \cap \cap_{i \in I} A_{2}$ is superfluous in $M$.

1.2. $\operatorname{crk}\left(M_{1} \oplus M_{2}\right)=\operatorname{crk}\left(M_{1}\right)+\operatorname{crk}\left(M_{2}\right)$ for any modules $M_{1}$ and $M_{2}$.

1.3. If $N$ is a submodule of $M$ then

$$
\operatorname{crk}(M / N) \leq \operatorname{crk}(M) \leq \operatorname{crk}(M / N)+\operatorname{crk}(N)
$$

This work was done while I was spending my sabbatical year at lzutgers University. I would like to thank the Mathematics Department at Rutgers, especially Professor Carl Faith, for their hospitality. 
and, when $\operatorname{crk}(M)$ is finite, $\operatorname{crk}(M / N)=\operatorname{crk}(M)$ if and only if $N$ is superfuous in $M$.

1.4. It follows from 1.3 and the exact sequence

$$
0 \rightarrow M /\left(N_{1} \cap N_{2}\right) \rightarrow\left(M / N_{1}\right) \oplus\left(M / N_{2}\right) \rightarrow M /\left(N_{1}+N_{2}\right) \rightarrow 0
$$

for submodules $N_{1}$ and $N_{2}$ of $M$ that if $\operatorname{crk}(M)$ is finite then

$$
\operatorname{crk}(M)-\operatorname{crk}\left(M /\left(N_{1}+N_{2}\right)\right) \leq \sum_{i=1}^{2}\left(\operatorname{crk}(M)-\operatorname{crk}\left(M / N_{i}\right)\right)
$$

Let now $R \subset S$ be a finite normalizing extension, write $S=\sum_{i=1}^{n} R a_{i}$ where $a_{i} R=R a_{i}$ for each $i, 1 \leq i \leq n$, and let ${ }_{s} M$ be a left $S$-module. We fix this notation throughout this article. It was shown in Bit-David and Robson [1] that

$$
\operatorname{rk}\left({ }_{s} M\right) \leq \operatorname{rk}\left({ }_{R} M\right) \leq n \cdot \operatorname{rk}\left({ }_{s} M\right) .
$$

Since the proof of the second inequality appeals to Zorn's lemma, it is not clear that a formal dual of this result holds true. The purpose of this note is to show that under certain conditions, the inequality

$$
\operatorname{crk}\left({ }_{R} M\right) \leq n \cdot \operatorname{crk}\left(s_{S} M\right)
$$

is valid.

2. If $a \in S$ is a normal clement of $S$, that is, if $R a=a R$, then for a submodule $N$ of ${ }_{R} M, a N$ is a submodule of ${ }_{R} M$. The map $K \mapsto a^{-1} K \cap$ $N=\{x \in N \mid a x \in K\}$ is a one-to-one function that takes a family of coindependent submodules of $R(a N)$ into a family of coindependent submodules of ${ }_{R} N$, so $\operatorname{crk}(a N) \leq \operatorname{crk}(N)$. If $a M=M$ and $a$ is not a zero divisor on $M$ then the map $K / N \mapsto a K / a N$ becomes a one-to-one finction that takes a coindependent family of $R$-submodules of $M / N$ to a coindependent family of submodules of $M / a N$. It follows that in this case, $\operatorname{crk}(M / a N) \geq \operatorname{crk}(M / N)$. We shall find it necessary to introduce the set $\mathcal{N}$ of all submodules ${ }_{R} N$ of ${ }_{R} M$ such that $S N=M$.

Lemma 2.1. If $N_{1}, \ldots, N_{k}$ is a coindependent family of submodules of ${ }_{R} M$ such that $S N_{i} \neq M$ for $i=1, \ldots, k$ then $S N_{1}, \ldots, S N_{k}$ is a coindependent family of submodules of $S M$.

Proof: This follows from the observation that $S N_{i}+\bigcap_{j \neq i} S N_{j} \supseteq$ $S\left(N_{i}+\bigcap_{j \neq i} N_{j}\right)$. 
Proposition 2.2. If $N$ is a minimal member of $\mathcal{N}$ then

$$
\operatorname{crk}\left({ }_{R} N\right) \leq \operatorname{crk}\left({ }_{S} M\right) \leq \operatorname{crk}\left({ }_{R} M\right) \leq n \cdot \operatorname{crk}\left({ }_{R} N\right) \leq n \cdot \operatorname{crk}\left({ }_{S} M\right) .
$$

In particular, $(*)$ is true when either of the modules ${ }_{R} M$ or ${ }_{S} M$ is artinian.

Proof: The first incquality follows from Lemma 2.1 and the minimality of $N$. Since $M$ is the homomorphic image of $\bigoplus_{i=1}^{n} a_{2} N$, we deduce from 1.3 and the remarks preceding Lemma 2.1 that $\operatorname{crk}_{R} M \leq n \cdot \operatorname{crk}_{R} N$. Note that by Lemanoire [4], $R M$ is artinian if and only $S M$ is artinian.

Corollary 2.3. If $S M$ is a module such that ${ }_{R} M$ has a submodule $N$ with $S \otimes_{R} N \cong{ }_{S}(S N)$ then $\operatorname{crk}\left({ }_{R} N\right) \leq \operatorname{crk}(S M)$. In particular, we have

(i) $\operatorname{crk}\left({ }_{R} R\right) \leq \operatorname{crk}(s S)$;

(ii) if $S \otimes_{R} M \cong{ }_{S} M$ then $\operatorname{crk}\left({ }_{R} M\right)=\operatorname{crk}\left({ }_{S} M\right)$.

Proof: If $K$ is a submodule of ${ }_{R} N$ then the hypothesis implies that $S \otimes_{R}(N / K) \cong S N / S K$. It follows from Shamsuddin [5] that $S K \neq M$ if $K \neq N$. Lemma 2.1 now gives the inequality $\operatorname{crk}\left({ }_{R} N\right) \leq \operatorname{crk}\left({ }_{S} M\right)$. Observe that $S \otimes_{R} R \cong{ }_{S} S$ and we always have $\operatorname{crk}(S M) \leq \operatorname{crk}\left({ }_{R} M\right)$, so the last two statements follow.

Proposition 2.4. Suppose that for each $i, 1 \leq i \leq n$ the group homomorphism $M \rightarrow a_{2} M$ given by $x \mapsto a_{i} x$ is an isomorphism. Then

$$
\operatorname{crk}(s M) \leq \operatorname{crk}(R M) \leq n \cdot \operatorname{crk}(s M) .
$$

Proof: We show first that if $\operatorname{crk}\left({ }_{S} M\right)$ is finite then so is $\operatorname{crk}\left({ }_{R} M\right)$. By induction on the integer $k, 1 \leq k \leq n$, we show that if ${ }_{R} M$ has an infinite coindependent family of submodules then there exists an infinite coindependent family $\left(M_{i}\right)_{i \in \mathbb{N}}$ of submodules of ${ }_{R} M$ such that the family $\left(\bigcap_{j=1}^{k} a_{j}^{-1} M_{i}\right)_{i \in \mathbb{N}}$ is coindependent. We may assume that $a_{1}=1$, so the base case of the induction is clear. Let $1 \leq k<n$ and assume that $\left(\bigcap_{j=1}^{k} a_{j}^{-1} M_{i}=T_{i}\right)_{i \in \mathbb{N}}$ is coindependent. Put $a=a_{k+1}$ and observe that $\left(a^{-1} M_{i}\right)_{i \in \mathbb{N}}$ is coindependent. If $a\left(\bigcap_{i=r}^{\infty} T_{i}\right)+\bigcap_{i=r}^{\infty} M_{i}=M$ for some $r \in \mathbb{N}$ then the family $\left(T_{i} \cap a^{-1} M_{i}\right)_{i \geq r}$ is coindependent and we are then done. Otherwise, $\mathbb{N}$ partitions into disjoint non-empty finite subsets $A_{i}$ such that for each $j \in \mathbb{N}, N_{j}=a\left(\bigcap_{i \in A_{j}} T_{i}\right)+\bigcap_{i \in A_{j}} M_{\imath}$ is a proper submodule of $M$ and so the family $\left(N_{j}\right)_{j \in \mathbb{N}}$ is coindependent. But $\bigcap_{i \in A_{j}} T_{i} \subseteq \bigcap_{2=1}^{k+1} a_{i}^{-1} N_{j}$ and because $\left(\bigcap_{i \in A_{j}} T_{i}\right)_{i \in \mathbb{N}}$ is coindependent, we conclude that $\left(\bigcap_{i=1}^{k+1} a_{i}^{-1} N_{j}\right)_{j \in \mathbb{N}}$ is also coindependent. Since 
the submodules $\bigcap_{i=1}^{n} a_{i}^{-1} M_{j}$ are actually $S$-submodules of $S M$, we deduce that $s M$ has infinite dual Goldie dimension.

Next we show that

$$
\operatorname{crk}\left({ }_{R} M\right) \leq n \cdot \operatorname{crk}(S M) .
$$

It is possible to choose a member $N \in \mathcal{N}$ such that $\operatorname{crk}(M / N)$ is as large as possible. By 1.1, there exists a family $H_{1}, \ldots, H_{r}$ of submodules of $M$ such that $N, H_{1}, \ldots, H_{r}$ is coindependent, $N \cap H_{1} \cap \cdots \cap H_{r}$ is superfluous in ${ }_{k} M$ and each $M / H_{i}$ is hollow. Since $M /\left(N \cap H_{i}\right) \cong M / N \oplus M / H_{i}$, we have $\operatorname{crk}\left(M /\left(N \cap H_{i}\right)\right)>\operatorname{crk}(M / N)$, hence $S\left(N \cap H_{i}\right) \neq\left(N \cap H_{i}\right)$. Lemma 2.1 now implies that $r \leq \mathrm{crk}(S M)$. It follows from 1.1 and 1.3 that $\operatorname{crk}\left({ }_{R} M\right)=\operatorname{crk}\left({ }_{R}(M / N)\right)+r$, so $\operatorname{crk}\left({ }_{R} M\right)-\operatorname{crk}\left({ }_{R}(M / N)\right) \leq$ $\operatorname{crk}(S M)$. Using 1.4 and the observation that $\operatorname{crk}\left(M / a_{2} N\right) \geq \operatorname{crk}(M / N)$ we now conclude that

$$
\operatorname{crk}\left({ }_{R} M\right) \leq \sum_{i=1}^{n}\left(\operatorname{crk}(M)-\operatorname{crk}\left(M /\left(a_{i} N\right)\right)\right) \leq n \cdot \operatorname{crk}(s M) .
$$

\section{References}

1. J. Bit-David AND J. C. RoBson, "Title book?" Lecture Notes in Mathematics 825, Springer-Verlag, Berlin, Now York, pp. 1-5.

2. P. Grzeszczuck and E. R. Puczylowski, On Goldie and dual Goldie dimensions, J. Pure and Appl. Alg. 31 (1984), 47 54.

3. A. Hanna and A. Shamsudin, Duality in the category of modules. Applications, Algebra Berichte 49 (1984).

4. B. Lemanoire, Dimension de Krull et codéviation. Application au theoreme d'Eakin, Communications in Algebra 6 (1978), 1647-1665.

5. A. Shamsuddin, Finite normalizing extensions, Jour. Alg. 151 (1992), 218220.

6. K. Varadarajan, Dual Goldie dimension, Comm. Alg. 7 (1979), $565-610$. 